\title{
New SHH and Known SIX3 Variants in a Series of Latin American Patients with Holoprosencephaly
}

\author{
Viviane Freitas de Castro ${ }^{a, b}$ Daniel Mattos ${ }^{a, b} \quad$ Flavia Martinez de Carvalho $^{b, c}$ \\ Denise Pontes Cavalcanti $^{d}$ Milagros M. Duenas-Roque ${ }^{e}$ Juan Llerena Jr $^{b, f}$ \\ Viviana Raquel Cosentino ${ }^{g}$ Rachel Sayuri Honjo ${ }^{h}$ Julio Cesar Loguercio Leite ${ }^{i}$ \\ Maria Teresa Sanseverino ${ }^{i}$ Márcia Pereira Alves de Souza ${ }^{j}$ Pricila Bernardik \\ Ana Maria Bolognesel Luiz Carlos Santana da Silva ${ }^{\mathrm{b}, \mathrm{m}}$ Pablo Barbero $^{\mathrm{n}}$ \\ Patricia Santana Correia ${ }^{f}$ Larissa Souza Mario Bueno $^{\circ}$ \\ Clarice Pagani Savastano ${ }^{a}$ lêda Maria Oriolia, b

\begin{abstract}
aECLAMC at Departamento de Genética, UFRJ, Rio de Janeiro, Brazil; 'blnstituto Nacional de Genética Médica Populacional INAGEMP, Porto Alegre, Brazil; 'ECLAMC at Laboratorio Epidemiol. Malformações Congênitas, IOC/FIOCRUZ, Rio de Janeiro, Brazil; ' Departamento de Medicina Translacional, área de Genética Médica, FCM/ UNICAMP, Campinas, Brazil; ' ECLAMC at Servicio de Genética, Hospital Nacional Edgardo Rebagliati Martins/

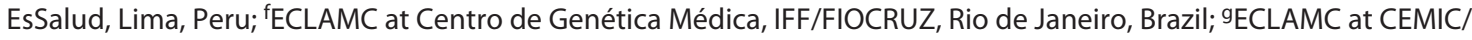
CONICET, Buenos Aires, Argentina; ' 'Unidade de Genética, HC/FM/USP, São Paulo, Brazil; 'ECLAMC at HC/UFRGS, Porto Alegre, Brazil; jUnidade Neonatal Nicola Albano, HUPE/UERJ, Rio de Janeiro, Brazil; ${ }^{k}$ Núcleo de Genética Clínica, Departamento de Clínica Médica/UFSC, Florianópolis, Brazil; 'Departamento de Ortodontia, Faculdade de Odontologia/UFRJ, Rio de Janeiro, Brazil; mLaboratório de Erros Inatos de Metabolismo, Instituto de Ciências

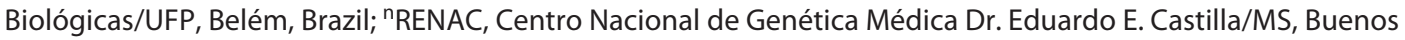
Aires, Argentina; ${ }^{\circ}$ Complexo Hospitalar Universitário Professor Edgard Santos, UFBA, Salvador, Brazil
\end{abstract}

\section{Keywords}

Holoprosencephaly · Latin American populations $\cdot \mathrm{SHH}$.

ZIC2 · SIX3 · TGIF1 · Synonymous variants

\begin{abstract}
Holoprosencephaly (HPE) is the failure of the embryonic forebrain to develop into 2 hemispheres promoting midline cerebral and facial defects. The wide phenotypic variability and causal heterogeneity make genetic counseling difficult.
\end{abstract}

Heterozygous variants with incomplete penetrance and variable expressivity in the SHH, SIX3, ZIC2, and TGIF1 genes explain $\sim 25 \%$ of the known causes of nonchromosomal HPE. We studied these 4 genes and clinically described 27 Latin American families presenting with nonchromosomal HPE. Three new SHH variants and a third known SIX3 likely pathogenic variant found by Sanger sequencing explained $15 \%$ of our cases. Genotype-phenotype correlation in these 4 families and published families with identical or similar driver gene, mutated domain, conservation of residue in other spe- karger@karger.com

(c) 2021 S. Karger AG, Basel

www.karger.com/msy

Karger'
Correspondence to:

Iêda Maria Orioli, ieda.orioli@gmail.com 
cies, and the type of variant explain the pathogenicity but not the phenotypic variability. Nine patients, including 2 with $\mathrm{SHH}$ pathogenic variants, presented benign variants of the $S H H, S I X 3, Z I C 2$, and TGIF1 genes with potential alteration of splicing, a causal proposition in need of further studies. Finding more families with the same SIX3 variant may allow further identification of genetic or environmental modifiers explaining its variable phenotypic expression.

(c) 2021 S. Karger AG, Basel

\section{Introduction}

Holoprosencephaly (HPE) is the most common central nervous system (CNS) defect in humans. The prevalence rate is $1 / 250$ conceptions [Matsunaga and Shiota, 1977]. In Latin America, the birth prevalence rate (live and stillbirths) was estimated at 1 per 10,000 [Orioli and Castilla, 2010]. This congenital brain malformation develops during the first month (between the 18th and the 28th day) of gestation and consists of incomplete cleavage of the forebrain into the cerebral hemispheres and subcortical structures along the CNS midline. The brain defects often result in midline facial anomalies and are classified into 3 types, from the most to the least severe: HPE alobar, semilobar and lobar, besides a milder subtype, the middle interhemispheric variant (MIHV). Generally, the severity of the facial defects is associated with the severity of the brain defect [DeMyer et al., 1964]; however, milder forms of facial anomalies may be present without brain malformation and then are called microforms such as the single median maxillary central incisor (SMMCI) or ocular hypotelorism [Cohen, 1989a; Richieri-Costa and Ribeiro, 2006].

Gross numeric and structural chromosomal anomalies cause $25-45 \%$ of the HPE cases. Variants in the genes sonic hedgehog $(\mathrm{SHH})$ [Roessler et al., 1996], zinc finger protein of the cerebellum 2 (ZIC2) [Brown et al., 1998], sine oculis homeobox homolog 3 (SIX3) [Wallis et al., 1999], and TG-interacting factor (TGIF1) [Gripp et al., 2000] correspond to $20-25 \%$ of nonchromosomal/nonsyndromic HPE cases [Mercier et al., 2011]. Small copy number variations $(\mathrm{CNV})$ and these single gene variants explain around 30-35\% of the HPE cases [Dubourg et al., 2018; Kim et al., 2019], leaving around 70\% of nonchromosomal/nonsyndromic HPE cases without a molecular diagnosis. Identifiable syndromes, variants in minor HPE genes, or more complex etiologies involving genetic and environmental factors also contribute to HPE etiologic heterogeneity [Cohen, 1989b; Roessler et al., 2012]. Few known environmental factors contribute to HPE com- plex etiology in humans as prenatal maternal exposure to diabetes, alcohol drinking, retinoic acid, and cholesterol deficiency [Cohen and Shiota, 2002; revised by Johnson and Rasmussen, 2010].

$\mathrm{SHH}$ was the first gene to be associated with HPE [Roessler et al., 1996] and even now remains the leading cause of nonchromosomal HPE [Dubourg et al., 2016]. $\mathrm{SHH}$ encodes a signaling protein with a key role in early embryonic development, such as the development of brain and midline facial structures [Belloni et al., 1996]. The Human Gene Mutation Database (HGMD ${ }^{\circledR}$; http:// www.hgmd.cf.ac.uk/; access date February 18, 2020) holds 163 disease-causing variants in the $\mathrm{SHH}$ gene associated with HPE, while ClinVar exhibits 36 pathogenic/ likely pathogenic variants (access date October 8, 2020) [Landrum et al., 2018]. The truncation variants are more clearly connected to the impaired signaling function of the $\mathrm{SHH}$ protein, while missense variants in both $\mathrm{SHH}-\mathrm{N}$, where they are more frequent, and $\mathrm{SHH}-\mathrm{C}$, indicate a role in $\mathrm{SHH}$ auto-catalytic processing throughout mechanisms not fully understood [revised by Roelink, 2018].

ZIC2 has been described as the second identified gene and the second most frequently mutated gene in HPE cases [Brown et al., 1998; Dubourg et al., 2007]. ZIC2 has several roles in neurological development, including the generation of neural crest, and it acts as a transcriptional activator or repressor. Through its zinc finger domain, ZIC2 is able to bind to DNA sequences that can also be bound by Gli proteins, which suggests the interaction of ZIC2 in the SHH signaling pathway [Aruga, 2004]. ZIC2 variants are linked to both classical and MIHV forms of HPE [Fernandes et al., 2007; Maurus and Harris, 2009]. In the MIHV, the error is in the dorsal neural patterning, unlike the classic forms of HPE where the error occurs in the ventral neural patterning. ZIC2 hypomorphic alleles interacting latter with the $\mathrm{SHH}$ pathway could cause the MIHV, although the precise ZIC2 molecular mechanisms are not well understood [reviewed by Barratt and Arkell, 2018].

The SIX3 gene plays a role in the development of the eyes and anterior forebrain during early vertebrate development. The SIX3 protein carries a DNA-binding homeobox domain and a SIX domain that can enlist factors to activate or repress other signaling pathways. The SIX3 transcription factor forms complexes with the members of general corepressor Groucho to repress BMP, Wnt, and Nodal targets during the ventral forebrain growth and patterning. SIX3 additional functions include to directly regulate $S H H$ expression, but also be regulated by $\mathrm{SHH}$ in a feedback loop, acting on critical factors to the 
ventral forebrain specification [revised by Domené et al., 2008 and Geng et al., 2016].

Human TGIF1 encodes a homeobox protein with several alternative splice variants. All variants contain the 3-prime end of exon 10 and all of exon 11 encoding the homeodomain and the carboxyl-termini of the protein [Hamid et al., 2008]. TGIF1 has several transcriptional repressor and corepressor roles in the retinoid and TGF $\beta /$ Nodal and can independently regulate the SHH signaling pathway during the ventral midline forebrain patterning. Wotton and Taniguchi [2018] revised the several proposed mechanisms of how TGIF1 variants can contribute to the HPE phenotype.

Several screenings for variants in patients with $\mathrm{HPE}$ gave an estimated rate of $8.2 \%$ in $\mathrm{SHH}, 7.4 \%$ in $\mathrm{ZIC2}, 3.9 \%$ in SIX3, and 1.1\% in TGIF1 [Mercier et al., 2011]. The low frequency initially described for TGIF1 variants in HPE patients, 1.5\% (95\% CI 0.4-3.8) [Gripp et al., 2000], did not change in more recent cohorts [Mercier et al., 2011; Roessler et al., 2012, 2018a; Dubourg et al., 2016]. TGIF1 has a minor HPE gene status nowadays [Dubourg et al., 2016; Roessler et al., 2018b] after other genes as GLI2 (\#165230), FGFR1 (\#136350), and FGF8 (\#600483) being recognized as often associated with HPE. Variants in these 3 genes are phenotypically polymorphic, being associated with several syndromes with or without HPE, for example, hypogonadotropic hypogonadism 2 with or without anosmia (\#147950), Culler-Jones (\#615849), Hartsfield (\#615465), and several skeletal dysplasias. So, their frequency in HPE cohorts will depend on how many patients with HPE or microforms have a complete or incomplete form of any of those syndromes. At least 10 other genes, considered minor HPE genes, were also described in HPE patients [Dubourg et al., 2016; revised by Roessler et al., 2018b]. The number of the genes potentially involved are growing due to next-generation sequencing, like the PPP1R12A gene recently associated with HPE and urogenital malformations [Hughes et al., 2020].

Most of the HPE-associated gene variants are related to autosomal dominant inheritance [Odent et al., 1998]. The same variant frequently occurs in fully affected, mildly affected, or a normal person of the same family, indicating a complex etiology. There are examples of recessive inheritance in HPE, including compound heterozygous variants or homozygosity [El-Jaick et al., 2007; McCabe et al., 2011], digenic, and oligogenic inheritance with mutations in 2 or more HPE genes [Mouden et al., 2016; Dubourg et al., 2016, 2018]. However, they have been, until now, rare mechanisms in HPE pathogenesis, and Roessler et al. [2012] showed that the "autosomal dominant with modifier" model better explained the HPE incomplete penetrance and variable expressivity [Roessler et al., 2018b]. Several models proposed in mouse, such as double haploinsufficiency, the interaction of a loss-of-function driver variant with a hypomorphic allele or silent allele, or with a background of modifiers, apply to human [Hong and Krauss, 2018]. Likewise, Roessler et al. [2018a] proposed it is important to consider co-occurring variants, both coding and noncoding, as it is sometimes possible to find evidence of potential gene-gene interactions that are consistent with a modifying effect. Such modifiers are context-dependent and can be silent without the presence of a driver variant [Roessler et al., 2018a].

New advances in studying HPE and other complex diseases to identify the genes acting to cause HPE in each family are those integrating genomic, epigenomic, transcriptomic, proteomic, phenomic, and metabolomic analysis [Kim et al., 2019; revised by Kerr et al., 2020]. Coherently with innovative approaches, Roessler et al. [2018a] said that "the best avenue to further our understanding of HPE lies in increasing numbers of cases rather than the number of genes considered."

In the present study, we aim to find variants of $\mathrm{SHH}$, ZIC2, SIX3, and TGIF1 genes that could explain the HPE in a Latin American cohort to enlarge the phenotypic and genetic HPE data and search for families with the same driver variant earlier published. We evaluated 27 families with probands presenting with HPE or SMMCI. The 4 classical HPE genes and clinical data were investigated in these families and the literature reviewed for similar genetic variants.

\section{Material and Methods}

The 27 probands with HPE or SMMCI came from Brazil (20), Peru (4), and Argentina (3). Fresh blood or DNA and the clinical information of the probands and relatives were obtained by collaborators of the Latin American Collaborative Study of Congenital Malformations (ECLAMC). ECLAMC is a hospital-based network aiming for collaborative research on congenital anomalies [Castilla and Orioli, 2004]. Familial clinical data received from the patients' physicians included physical examination, genealogical information, prenatal maternal teratogenic exposures, brain imaging, laboratory results, and informed consent. The proband exclusion criterion was chromosomal abnormalities confirmed by a conventional karyotype. There is no karyotype information for 5 of the 6 patients with SMMCI. No patients, but one, were studied for CNV. We included probands with HPE found by prenatal ultrasound, CT scan, MRI, autopsy reports, and those with facial characteristics of HPE or SMMCI. 
DNA Extraction and Sequencing

DNA was extracted by using salting out method [Miller et al., 1988]. Double strands of the coding regions and exon-intron borders of SHH, ZIC2, SIX3, and TGIF1 genes were submitted to Sanger sequencing. Amplification protocols of these regions and primers were described in Savastano et al. [2014]. Amplicons were visualized in 1\% agarose gel and further purified by GFX PCR DNA and Gel Band Purification kit (Ge Healthcare, UK), according to the manufacturer's instructions. Fragments were then sequenced using the Big Dye Terminator v3.1 Cycle Sequencing Kit (Applied Biosystems, Foster City, CA, USA). Products were sequenced at ABI Prism 3130xL (Applied Biosystems). Found variants were confirmed by independent experiments. Sequence data were analyzed using ChromasPro software 1.41 (Technelysium ${ }^{\circledR}$ ). Genomic, exonic, and protein sequences were downloaded from the National Center for Biotechnology Information (NCBI; https://www.ncbi.nlm.nih.gov/) using the hg19 genome version (online suppl. Table 1; for all online suppl. material, see www.karger.com/doi/10.1159/000515044).

\section{Pathogenicity Prediction}

To determine the pathogenicity status of all variants found, they were submitted to VarSome (https://varsome.com/), where they were classified according to ACMG criteria, and allele frequencies were annotated from the Genome Aggregation Database (gnomAD Exomes v.2.1.1). Variants were surveyed if already published in HGMD, and only those not found were considered new variants. Variants were evaluated at Human Splicing Finder (HSF; http://www.umd.be/HSF/index.html, access date February 18, 2020) for signals of impact on splicing cis elements that could alter the transcript [Ohno et al., 2018].

\section{Clinical Presentations and Results}

\section{General Clinical Information}

In this Latin American series, there were 3 fetuses, 7 newborns, and 17 patients with ages below 15 years. Figure 1 shows the 20 facial images available. Table 1 shows the country and the clinical features of the 27 probands. Twenty-one patients were ascertained due to a cerebral defect and 6 due to SMMCI. Only 1 patient presented with cebocephaly with more severe facial alterations, as cyclopia or ethmocephaly, and did not appear in this series. With or without brain defects, all patients showed developmental delay and/or intellectual disabilities, except the 4-month-old patient, who was not tested.

\section{Patients with SHH, ZIC2, SIX3, and TGIF1 Likely \\ Pathogenic Variants \\ Patient 1}

A 6-year-old male was born as the first child of a healthy 27-year-old mother and her 42-year-old nonconsanguineous healthy husband. The proband had mild microcephaly, right cleft lip and palate, and SMMCI. Cerebral images were not available, and he presented severe learning difficulties with preserved social skills (Table 1; Fig. 1). The proband presented a de novo likely pathogenic $S H H$ missense new variant (c.437T > G; p.Val146Gly) (Table 2; Fig. 2). He also presented a synonymous single nucleotide variant (sSNV) in SIX3 (rs78018362; p. Ala30=; c.90G $>$ T), and 3 benign TGIF1 variants: in the $5^{\prime}$ UTR (rs23813; c. $-33 \mathrm{C}>\mathrm{A}$ ), a synonymous variant (rs2229337; p.Pro140=; c.420A >G), and a missense variant (rs4468717; p.Pro163Ser; c.487C >T). The proband's mother also presented these 4 variants, and his half-sister only showed the 3 TGIF1 variants (Table 2; Fig. 2). HSF suggested the SIX3 synonymous variant rs78018362, with $0.88-9.32 \%$ population frequency, potentially produces an alteration of splicing with the creation of an exonic splicing silencer site and change of an exonic splicing enhancer (ESE) site. The missense TGIF1 variant was predicted to have no impact on splicing.

\section{Patient 2}

A 1-year-old male was born at term as the first child of a healthy 23-year-old mother and her healthy 26-year-old nonconsanguineous husband. The proband had microcephaly, ocular hypotelorism, a flat nose, and corrected premaxillary agenesis (Table 1). Cerebral CT showed a MIHV-HPE and corpus callosum hypoplasia. He presented with a new likely pathogenic small $\mathrm{SHH}$ deletion (c.329_337delCCATCTCGG; p.Ala110_Ser112del), and these 9-bp in-frame deletion induces the loss of 3 amino acids (alanine, isoleucine, and serine) in the polypeptide chain (Table 2; Fig. 2). The proband also presented a benign ZIC2 in-frame duplication (c.716_718dupACC; p.His239dup) (Table 2; Fig. 2). This small duplication expands the tract from 9 to 10 histidines as previously reported in control individuals [Brown et al., 2001]. HSF estimated this small in-frame duplication, with a population frequency of $0.07-12.70 \%$, potentially produces an alteration of splicing with the creation of an ESE site. Parents' DNA was not available.

\section{Patient 3}

A 40-week-old male neonate was born as the first child of a nonconsanguineous 23-year-old father and mother. The proband had microcephaly, premaxillary agenesis, ocular hypotelorism, and bilateral exophthalmia. The cerebral MRI showed semilobar HPE (Table 1). A new likely pathogenic $S H H$ missense variant (c.1088G > T; p.Cys363Phe) (Table 2; Fig. 2) was detected in the proband and his sister and mother, both affected with milder craniofacial anomalies. Both children, but not their mother, also carried a ZIC2 synony- 


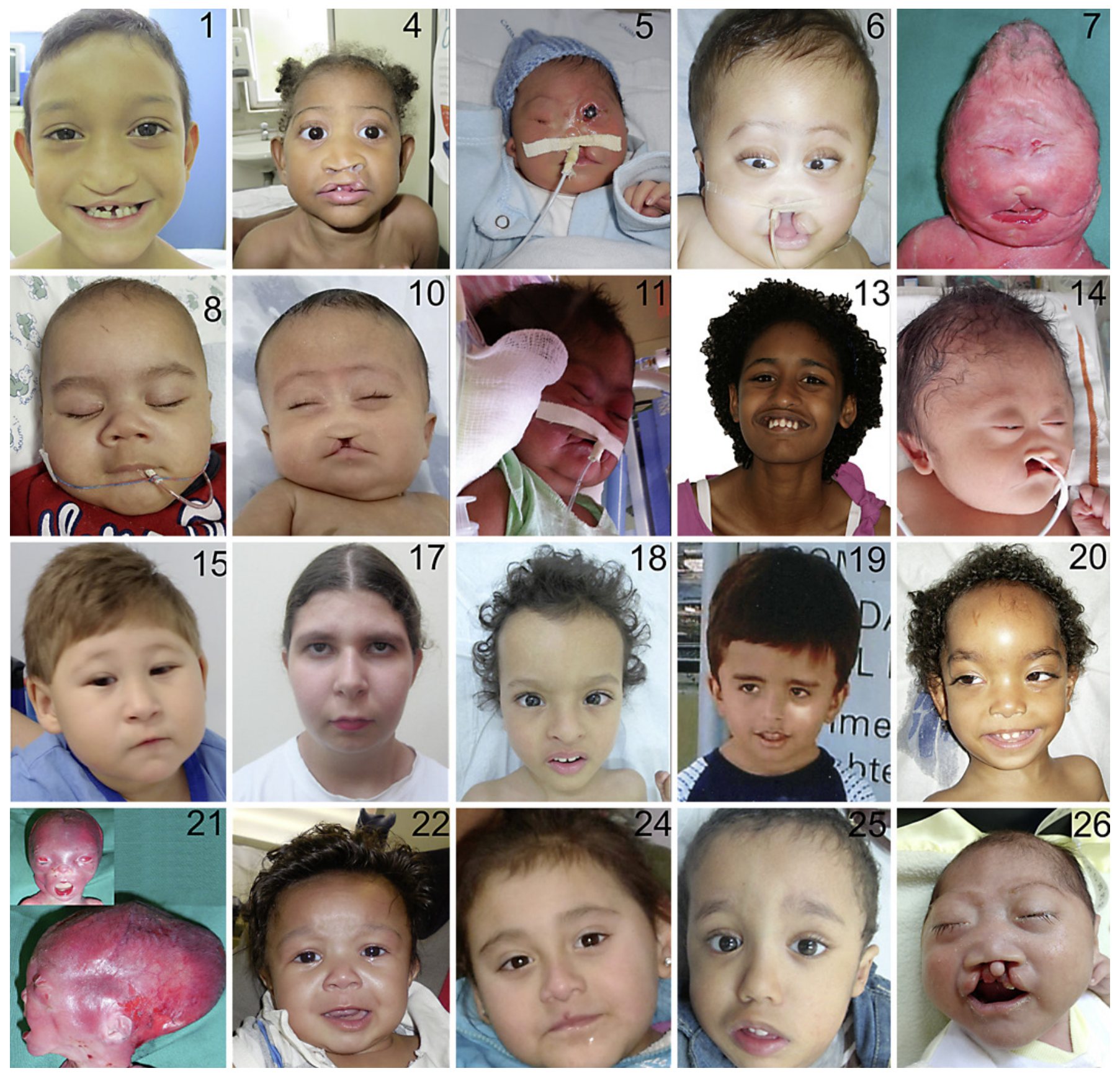

Fig. 1. The craniofacial aspect of 20 holoprosencephaly (HPE) probands in this series. Patients 2, 3, 9, 23, and 27 are not shown. Patients $8,13,24,25$, and 27 with single median maxillary central incisor had no brain defect, and the information was not available for patient 1. Among the HPE group, all the frequent HPE types were diagnosed: 7 alobar HPE in patient 5, 6, 10, 11, 14, 16, and 18; four semilobar in patients $3,9,20$, and 22; five lobar in patients 4 , $12,17,21$, and 26; and 2 middle interhemispheric variants in pa- tients 2 and 19; in the remaining 2 patients 7 and 23, the HPE type was not further specified. Patient 15 presented only with corpus callosum agenesis. Twelve patients presented with premaxillary agenesis $(2,3,5,6,10,11$, and 14$)$ or cleft lip and palate $(1,4,7,16$, and 26). Fourteen patients showed the absence of facial cleft $(8,9$, $12,13,15,17-22,24,25$, and 27). Microphthalmia was described in patients $5,6,7,23$, and 27 . 


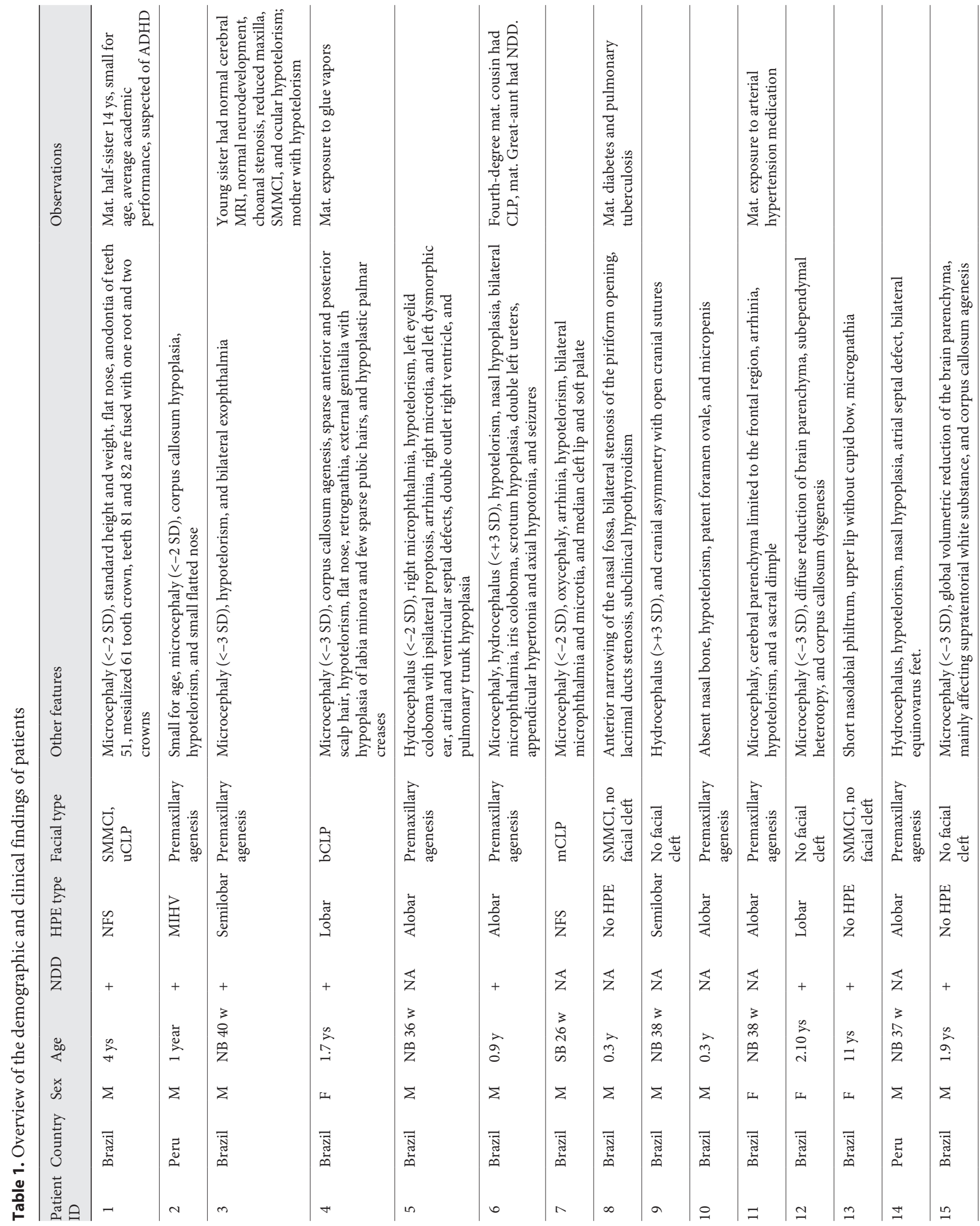




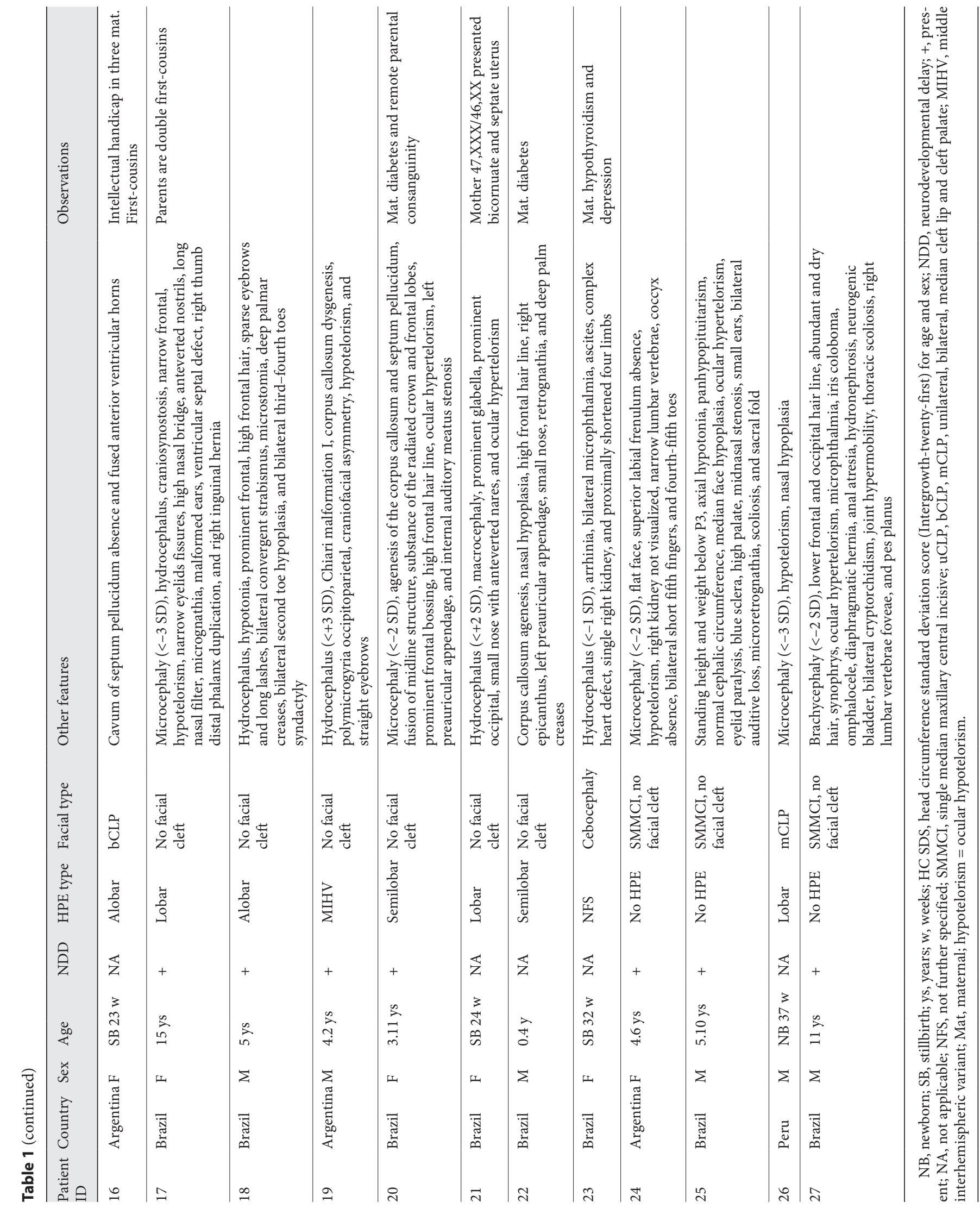




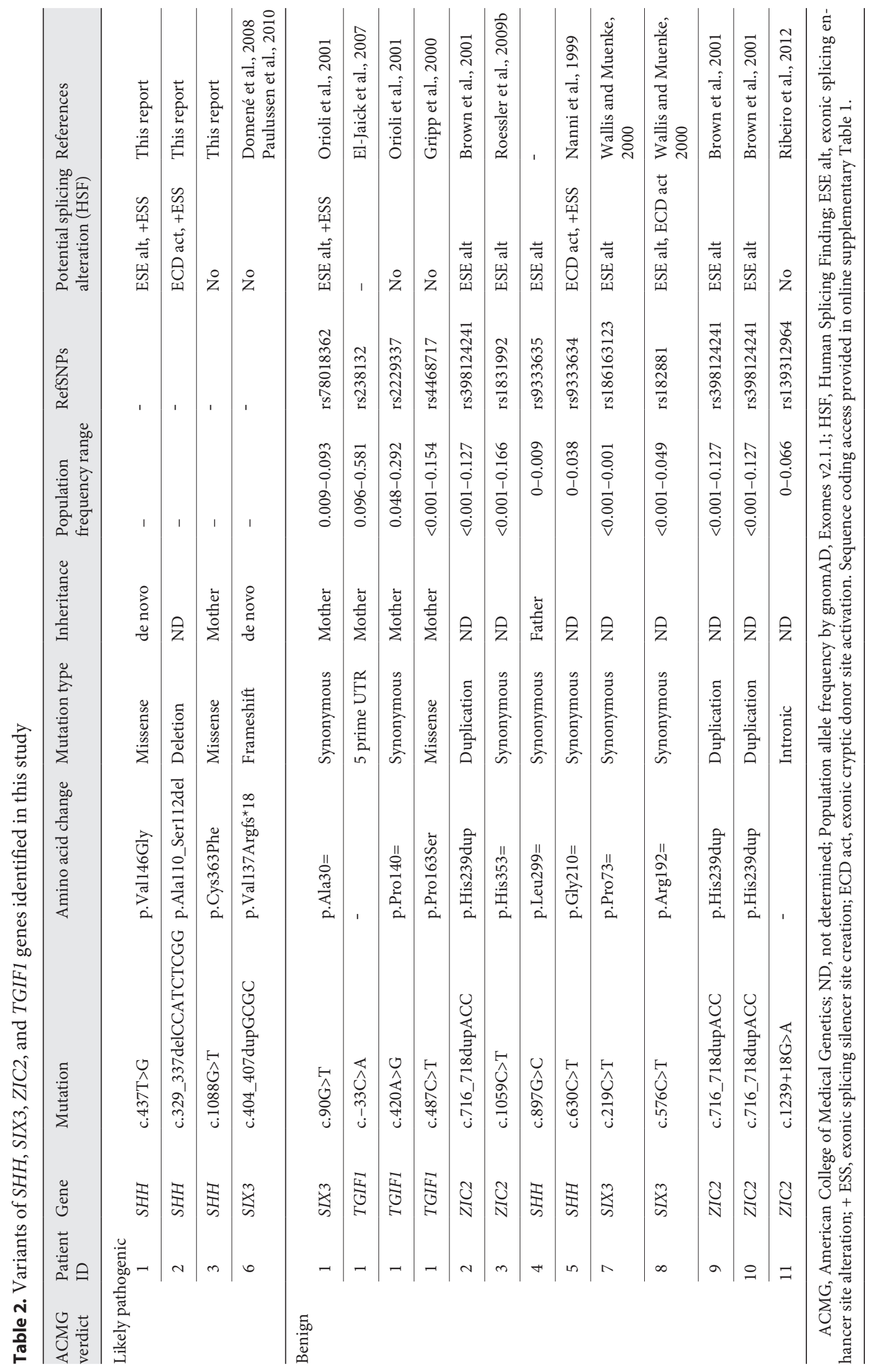




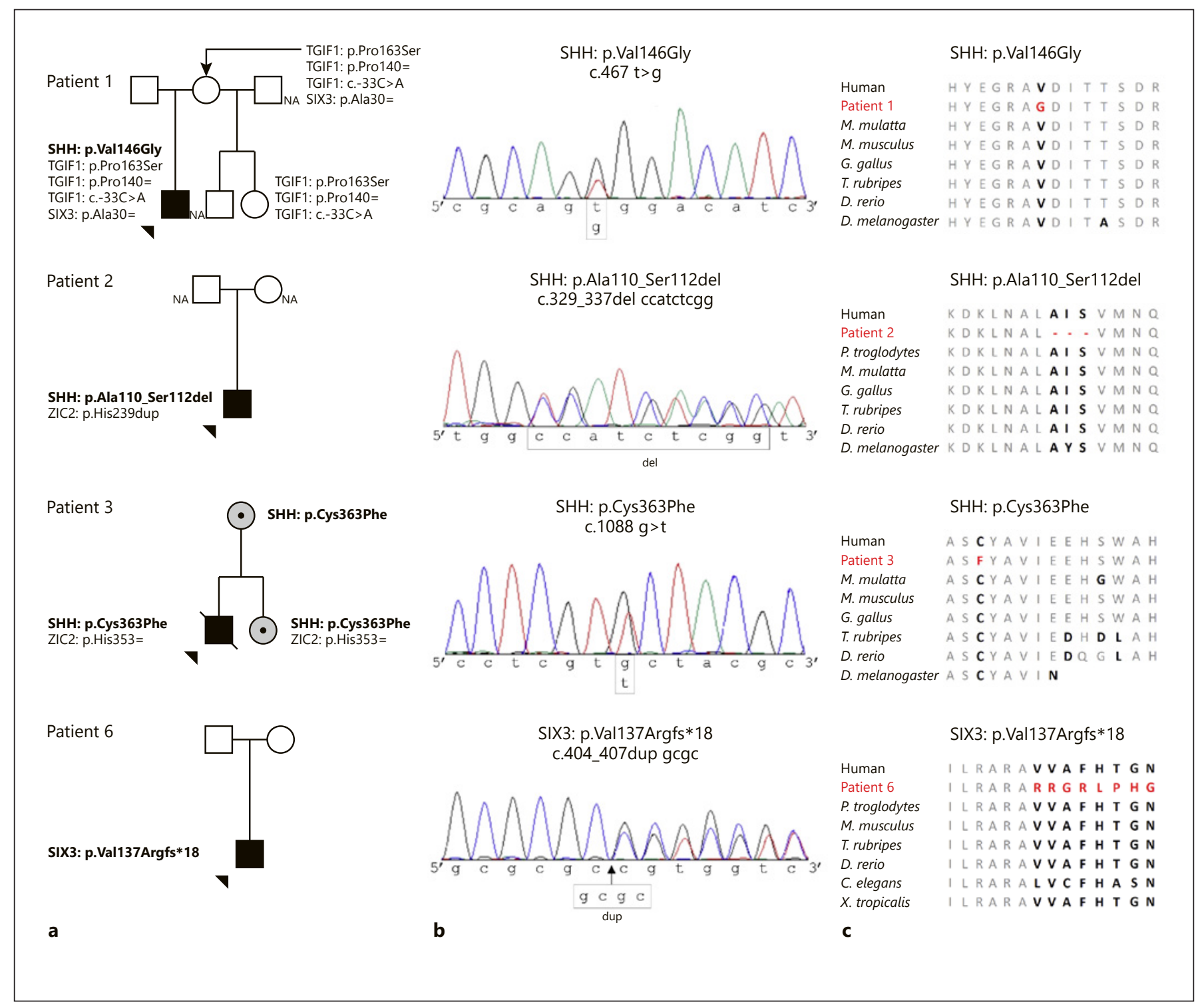

Fig. 2. a Pedigrees of 4 patients with HPE demonstrating the segregation of different SHH, SIX3, ZIC2 and TGIF1 variants with phenotypes. Black: fully affected. Gray: only microsigns. Black dot: carrier of pathogenic variants. NA: not available for molecular study. b Electropherograms of the 4 pathogenic variants. c Phylogenetic conservation of the amino acid sequence showing the position of the pathogenic variants in red and the conserved sequence in gray.

mous variant (rs1831992; c.1059C $>\mathrm{T}$; p.His353=) (Table 2; Fig. 2). HSF estimated this ZIC2 synonymous variant, with a $0.03-16.6 \%$ population frequency, potentially produces an alteration of splicing with the creation of an ESE site.

\section{Patient 6}

A 15-month-old male was born as the first child of a healthy 16 -year-old mother and her 20-year-old noncon- sanguineous healthy husband. The proband had alobar HPE diagnosed by prenatal ultrasound. He also had ocular hypotelorism, premaxillary agenesis, choanal atresia, bilateral microphthalmia, iris coloboma, and non-craniofacial defects (Table 1; Fig. 1). He had a de novo variant at SIX3, p.(Val137Argfs*18). This variant was a duplication of 4 bp at exon 1 of SIX3 (c.404_407dupGCGC) (Table 2; Fig. 2), which causes a frameshift at residue 137 and an early stop codon. 


\section{Patients with Only Benign Variants}

There were 4 exonic synonymous variants in 7 patients, 1 exonic codon duplication, and 1 intronic variant (Table 2). These variants were predicted as benign, but they may have a potential role in HPE due to the possibility of impacting splicing as estimated by HSF. The clinical data and variants of patients $4,5,7,8,9,10$, and 11 were shown in Figure 1, Tables 1 and 2, and online supplementary Table 2.

\section{Discussion}

\section{Cohort Characteristics}

We observed some accepted correlations among variants of SHH and SIX3 and the HPE phenotype in our series. The 3 patients with $S H H$ variants presented less severe HPE and relatively less-affected facial features than the patient with SIX3 variants, who also had severe eye defects. However, due to the small number of patients (27), the presence or absence of these characteristics has no statistical significance. The phenotype-genotype correlations in HPE were confirmed in large cohorts by Solomon et al. [2010], Mercier et al. [2011], and Dubourg et al. [2016]. Consistent correlations were the higher prevalence of facial anomalies in patients with $\mathrm{SHH}$ variants [Solomon et al., 2012], contrasting with no significant facial changes in those with ZIC2 variants [Solomon et al., 2010]. More severe HPE was predominant in patients with variants in $Z I C 2$ and $S I X 3$, and $S H H$ variants were associated with the milder cases or microforms. Keaton et al. [2010] described a wide range of clinical severity in patients with TGIF1 or SHH variants, showing less severe phenotypes than those observed for either the ZIC2 or SIX3 genes. Mercier et al. [2011] noted that alobar HPE with severe facial features seems to be more frequent in patients with TGIF1 variants and suggested to reanalyze in larger series.

Considering the known phenotype-genotype correlations, we found 2 inconsistencies in one patient (2) with MIHV. He presented premaxillary agenesis and a pathogenic variant in the $\mathrm{SHH}$ gene. It is more frequent to find a ZIC2 pathogenic variant and a mildly affected face in patients with MIHV [Mercier et al., 2011]. Besides the SHH variant, this patient had a ZIC2 histidine tract expansion predicting changes in the ESE site. A ZIC2 variant effect would be more in line with the presence of MIHV, although other genes not studied here have been described in patients with MIHV, such as FGF8 [Hong et al., 2018]. The other patient (19) with MIHV in our series was without facial dysmorphias, type 4 [Mercier et al., 2011], as expected, and showed polymicrogyria, a defect involving neuronal migration also correlated with ZIC2 variants [Solomon et al., 2010; Mercier et al., 2011]. However, we did not find any variant or environmental teratogen in patient 19 .

Another common characteristic of the HPE cohorts is the female-biased sex ratio, mainly involving the ZIC2 and SIX3 variants [Mercier et al., 2011]. Recently, Kruszka et al. [2019] showed 33 HPE cases associated with loss-of-function X-linked cohesin complex variants, of which 32 were females due to potential male lethality from these variants. Those 32 patients had less severe facial types (3 or 4) [Mercier et al., 2011], pointing to a particular subgroup of HPE patients. We found that only ethmocephaly, cebocephaly, and premaxillary agenesis were the facial types associated with the female-biased sex ratio in an earlier Latin American HPE cohort of 195 patients [Savastano et al., 2014]. There is no female excess in the current series with 17 males and 10 female patients, and patients had less severe or normal faces with only 1 patient with cebocephaly and 7 with premaxillary-agenesis, as observed before [Savastano et al., 2014].

\section{Pathogenic Variants and Phenotypes}

The variants found in patients 1 and 2, p.(Val146Gly) and p.(Ala110_Ser112del), were located toward the $S H H$ $\mathrm{N}$-terminal signaling domain, while the variant in patient 3 was in the SHH-C. This fact endorses the current idea that variants in structurally relevant modules in both the $\mathrm{N}$ - and C-terminal domain of $\mathrm{SHH}$ can prevent the processing of SHH pro-protein causing HPE [Traiffort et al., 2004; Roessler et al., 2009b; revised by Roelink, 2018].

Patient 1 exhibited a de novo variant in $\mathrm{SHH}$ (c.437T >G; p.Val146Gly) involving the Val-146 residue, close to the Asp-147 residue, one of the $\mathrm{Zn}^{2+}$ binding site coordinating residues in the $\mathrm{SHH} \mathrm{N}$-domain. The other coordinating residues are His-140, Glu-176, and His-182. Traiffort et al., [2004], in their functional study to characterize $\mathrm{SHH}$ variants, proposed the proximity of their mutated Thr-150 residue, p.(Thr150Arg), to the Asp-147 to explain the impaired production of the active SHH N-fragment. The mutated Val-146 of our patient 1 was in closer proximity to the Asp-147 residues, probably resulting in loose protein-protein interactions in some SHH N-complexes with its partners, but not in others, as proposed by Bosanac et al. [2009] for variants in the $\mathrm{Zn}^{2+}$ binding site coordinating resi- 
dues. So, this uncertain negative effect of those variants around the $\mathrm{ZN}^{2+}$ coordinating residues [Bosanac et al., 2009] could be related to the mild defects in patient 1 , as microcephaly ( $-2 \mathrm{SD}$ ) cleft lip, SMMCI, and intellectual disabilities. The patient with the variant in Asp150Thr [Traiffort et al., 2004] had classical HPE, described by Dubourg et al. [2003] with semilobar HPE, microcephaly, hypotelorism, cerebellar hypoplasia, and diabetes insipidus. It would be interesting to know the patient's phenotype with the p.(Asp147Arg) variant, but a further phenotypic description of the patient is not available [Roessler et al., 2009a].

Patient 1 , his mother, and the half-sister bore altogether 4 different nonpathogenic variants in SIX3 and TGIF1, 4 in the proband, besides the pathogenic $\mathrm{SHH}$ missense de novo variant. Clinically, only the patient presented a mild neuropsychological development delay with mild defects of the HPE spectrum. His half-sister was small for her age, without information about hypophyseal studies, and suspected of ADHD. The mother's intellectual abilities were not tested, and the cerebral images of the proband and half-sister were not available, which hindered a more specific genotype-phenotype correlation in this family. The 3 TGIF1 variants present in the proband, mother and maternal half-sister, a missense, a 5'UTR, and a synonymous, were described as common by ElJaick et al. [2007]. Also, El-Jaick et al. [2007] did functional studies on TGIF1 variants, including a missense variant, p.(Ser162Phe), located near the variant p. (Pro163Ser) found in the patient 1 family. The El-Jaick et al. [2007] patient had encephalocele, corpus callosum agenesis, median cleft lip, and inherited a paternal p.Ser$162 \mathrm{Phe}$ variant, whose protein performed normal in the functional studies.

Among the proband's 4 nonpathogenic variants, the synonymous SIX3 p.Ala30 = could potentially create an exonic splicing silencer site and change an ESE site. This variant mean frequency in the general population is 3.5\%, which is found in less than $1.5 \%$ of Europeans and Africans, 2 important components of the three-hybrid Latin American population. Only the proband had the SIX3 p.Ala30 $=$ synonymous variant combined with de novo SHH c.437T>G; p.Val146Gly variant, which could reinforce the pathogenicity of this last variant.

Regarding the SHH small in-frame deletion, c.329_337delCCATCTCGG; p.Ala110_Ser112del, found in patient 2 , a point variant generating a stop codon in the same region (c.335C $>$ A; p.Ser 112*) was described by Roessler et al. [2012]. This fact would indicate that not only the truncation of the protein at this site is patho- genic, but also the deletion of these 3 amino acid residues (Ala-110, Iso-111, and Ser-112). Patient 2 variant is not present in HGMD; however, other 6 different published missense variants involve these 3 residues [Nanni et al., 2001; Dubourg et al., 2004; El-Jaick et al., 2005; Roessler et al., 2009b, 2012]. Indeed, the SHH-N large alfa helix opposite to the $\mathrm{Zn}^{2+}$ coordination domain, from residue 100 to 117, showed 11 different variants found in holoprosencephalic patients [Traiffort et al., 2004; revised by Roelink, 2018]. Three different variants were tested in this region, 2 of which at residue Trp-117 [Maity et al., 2005] impaired the autocatalytic processing reaction, and 1 at residue Ile-111 [Singh et al., 2009] which decreased stability or secretion of $\mathrm{SHH}-\mathrm{N}$ protein. Only the stop codon variant p.(Ser112*) [Roessler et al., 2012] seems incompatible with the complete protein and justifies the severe phenotype alteration, as the patient with this variant exhibited cyclopia. The 3 codon deletions and other missense variants at these residues showed variable phenotypic alterations from just SMMCI in $\mathrm{p}$. (Ile111Phe) [Nanni et al., 2001] or corpus callosum agenesis in p.(Ala110Asp) [Dubourg et al., 2004] to MIHV with premaxillary agenesis or alobar HPE with premaxillary agenesis seen in our patient 2, p.(Ala110_Ser112del), and the patient described by El-Jaick et al. [2005], respectively.

The variant in $S H H, c .1088 \mathrm{G}>\mathrm{T}$, found in patient 3 changed a cysteine to a phenylalanine residue ( $\mathrm{p}$. Cys363Phe) in the C-terminal domain. Richieri-Costa and Ribeiro [2006] and Kruszka et al. [2015] reported a similar variant but changing Cys-363 to a tyrosine residue (c.1088 G>A; p.Cys363Tyr). Both substituted the small cysteine with larger amino acids, potentially disrupting the protein conformation at this site. Ser-362, Cys-363, and Tyr-364 (Fig. 2) were among the highly conserved residues in the intein-like elements [Roessler et al., 2009a], and 3 additional variants, c.1085C $>\mathrm{A}$ (p.Ser362*); c.1085C $>$ A (p.Ser362Leu), and c.1091A $>$ G (p.Tyr364Cys) were described by Roessler et al. [2009b] and Solomon et al. [2012], the first one in a patient with alobar HPE, and the others in patients with microcephaly and other minor HPE signs.

Our patient 3 ( $S H H$ p.Cys363Phe) had semilobar HPE with premaxillary agenesis similar to the patient published by Kruszka et al. [2015], p.(Cys363Tyr), but different from the patient published by Richieri-Costa and Ribeiro [2006], also with the p.Cys363Tyr variant, who presented an HPE-like face, a cyst of the choroidal fissure, and a normal neuropsychological evaluation. All of these 5 patients had affected relatives with minor HPE forms, 
like our patient 3 , showing that the wide intra- and interfamilial phenotypic variability observed is not constrained by the high phylogenetic conservation of the Ser362, Cys-363, and Tyr-364 residues. Also, we can note that the most severe case occurred in a truncation variant as expected [Solomon et al., 2012].

The last pathogenic variant identified in this HPE series was a de novo small duplication (c.404_407dupGCGC; p.Val137Argfs*18) that occurred inside 6 GC repeats of the SIX-domain of the SIX3 gene. Ribeiro et al. [2006] (c.406_407dupGC; p.Val137Profs*115) and Domené et al. [2008] (c.404_407dupGCGC; p.Val137Argfs*18 and c.405_409dupCGCCG; p.Val137Alafs*116) had already identified 3 truncation variants in this region, with duplication of 1 GC, 2 GC, and a CGCCG duplication. Our patient 6 belongs to the third family described with the SIX3 c.404_407dupGCGC; p.Val137Argfs*18 variant; the second family described by Paulussen et al. [2010]. These 3 frameshift variants changed the Val-137 residue producing a stop codon after the next 18 residues, as in our patient 6 , or after the 115 or 116 residues as in the other 2 duplications. Domené et al. [2008] did functional studies using zebrafish assays on these 3 frameshift variants showing that truncated SIX3 proteins lacking DNA-binding site could yet retain some repressive activity. They also showed that all 3 variants affected the SIX3 ability to repress BMD but not to equally repress Wnt [Domené et al., 2008]. Despite the observed functional differences, where the c.406_407dupGC, Val137Profs*115 was more severe than the GCGC or CGCCG duplications, because its mutants lost the capacity to repress both Wnt and BMD signaling, the correlation with the patients' phenotype is not so clear. The patient described by Ribeiro et al. [2006] with dup GC showed the same phenotype as in our patient 6 with dup GCGC, both presenting alobar HPE and premaxillary agenesis, while the patients described by Paulussen et al. [2010] and by Lacbawan et al. [2009] (the same case studied by Domené et al. [2008]) with c.405_409dupCGCCG variants presented semilobar HPE. Furthermore, the variant causing the most severe loss of function (c.406_407dupGC, Val137Profs*115), reported by Ribeiro et al. [2006], was present in the patient's father and paternal grandmother, both nonaffected individuals, disclosing further clinical variability. These 3 persons also presented a SIX3 variant (c.206G>A; pGly69Asp), tested near-normal functional activity [Domené et al., 2008]. Analyzing the diverse ways SIX3 participates in the ventral telencephalon specification, Geng et al. [2016] showed in a mouse model that different reduction in SIX3 dosage causes different HPE types, as alobar, lobar, semilobar or normal brain. According to the disclosed ways of SIX3 action, a variant in SHH, PATCHED, FOXG1, or in the SIX3 regulatory region, added to the SIX3 variant, must result in different human HPE forms [Geng et al., 2016]. Additional variants in any of these modifiers could explain the SIX3 functional studies differences between the observed phenotype and the expected. Then, further study of those 5 families with SIX 3 variants inside the GC repeats of the SIX-domain using integrated -omics analysis [Kim et al., 2019; Kerr et al., 2020], followed by proper allele-specific functional analysis [Domené et al., 2008; Hong et al., 2016; Soukarieh et al., 2016; Kim et al., 2020] could help to increase our understanding of HPE genes pathogenesis.

\section{Nonpathogenic Variants}

Pathogenicity status of variants follows strict rules to not include false-positive variants in curated databases, which could lead to false molecular diagnostics and wrong genetic counseling. We followed ACMG guidelines to classify variants. However, knowledge about variants' impact is still growing, and pathogenicity assigning algorithms may elude potentially pathogenic variants [Hong et al., 2017; Kim et al., 2019]. The exclusion of variants with lesser probabilities of pathogenicity creates a bias toward continuously excluding them from publications. For these reasons, we decided to report 13 variants classified as benign that occurred in 11/27 patients. Seven of these nonpathogenic variants are synonymous, and the others a missense, small duplication, $5^{\prime} \mathrm{UTR}$, or intron variant.

Chen et al. [2010] did a broad survey of single nucleotide variants (SNVs) reviewing genome-wide association studies. They found that nonsynonymous SNV (nsSNV) and synonymous SNV (sSNV) exhibited similar likelihood and effect size for disease association. Although acknowledging that these sSNVs might not be causal, they argue that these sSNVS are not likely to be in linkage disequilibrium with another nsSNV and that they are highly likely to be implicated in the disease process [Chen et al., 2010]. sSNV may affect many molecular aspects that could lead to pathogenic dysfunctional proteins. sSNV can disturb transcription as $\sim 15 \%$ of human codons simultaneously code for amino acids and transcription factor recognition sites [Stergachis et al., 2013]. They can also alter the expected splicing as exemplified by Pagani et al. [2005] that a quarter of all sSNVs tested resulted in exon 12 skipping in the CFTR protein. sSNVs may also interfere with mRNA secondary structure and stability. Presnyak et al. [2015], studying yeast, showed that codon optimality (the advantage of a codon over another that codes for the same amino acid) contributes to mRNA sta- 
bility and thus to fine-tuning the levels of gene expression due to variations in the half-life of transcripts with different sSNV. Codon optimality may also interfere in the rate of elongation during translation in yeast and thus on the early protein stability [Pechmann and Frydman, 2013].

In a recent study, Kim et al. [2020] found sSNV enriched in patients with HPE compared to healthy individual cohorts. Further investigating these sSNV, the authors found no evidence of their impact on splicing by both in silico and in vitro assays. However, 5 sSNV predicted by in silico analyses to impact the translation of $\mathrm{SHH}$ were also confirmed by in vitro assays to result in a decrease of the SHH protein quantity. Furthermore, they presented evidence that this reduction in protein is due to selective degradation by the proteasome. In our series, families 4 and 5 carried 2 of the 5 sSNV studied by Kim et al. [2020]. SHH c.897G $>$ C, seen in family 4, reduced the SHH protein by $13 \%$, and $S H H$ c.630C $>$ T, observed in family 5 , reduced the protein by $5 \%$. Both our patients were severely affected, and their cephalic and extracephalic defects have been described in HPE cohorts [Martinez et al., 2018]. To detect sSNV with potential splicing effects, we have used HSF, a bioinformatic tool that was qualified by experimental studies as the minigene splicing assay [Soukarieh et al., 2016]. Our inspection of the mRNA structure of $S H H$ c.897G $>$ C variant compared with wild mRNA using VARNA (http://varna.lri.fr/) [Darty et al., 2009] revealed structural modifications. However, Kim et al. [2020] inspecting the optimal mRNA structure and base-pairing probabilities did not predict any changes. Then, our preliminary information about the variants not tested by Kim et al. [2020] requires further research to show the effective involvement of these sSNVs in HPE causality, especially functional studies like the splicing minigene reporter assay [Soukarieh et al., 2016; Kim et al., 2020]. In the future, it will need confirmation that the results from the minigene context are the same in the patient cellular and genomic context [Fu and Ares, 2014]. In the series presented here, we could not exclude that patients only carrying benign variants also had a loss-of-function variant in HPE genes that were not studied here.

\section{Conclusion}

We studied the SHH, ZIC2, SIX3, and TGIF1 genes in a Latin American series of 27 patients. We found 4 families with 3 novel variants in $\mathrm{SHH}$ and a third family with the same published SIX3 frameshift variant, explaining
$15 \%$ of our cases. Recent literature shows that the classical 4 genes stay an important source of rare variants. There are novel genes implicated in HPE causality, mainly as modifiers that must be studied in negative or inconclusive cases. The third family we found with a SIX3 rare variant (p.Val137Argfs*18) deserves a collaborative study including all similar families previously published. We suggest observing the phenotype-genotype correlations based on NGS results and also from other -omics tools in families with the same driver variant as a productive approach to our understanding of HPE pathogenesis. Nine patients, including 2 with $S H H$ pathogenic variants, presented benign variants with potential alteration of splicing, a causal proposition in need of further studies. The role of splicing errors from synonymous or other benign variants causing human diseases is well known in the medical literature. However, HPE literature frequently overlooked those variants.

\section{Acknowledgments}

We thank the patients and their families for participating in the study and all physicians who provided us with the patients' data. We are grateful to Maura Silva and Kelli Mendes from UFRJ; Hector Seuanez, Miguel Moreira, Bruna Matta, and Renata Olicio from INCa for technical support, and Ana Carolina Fonseca and Leandro Landeira for their valuable contribution. This study is part of V.F. de Castro's thesis research in pursuit of her PhD in Genetics at the Genetics Department of Federal University of Rio de Janeiro.

\section{Statement of Ethics}

The study was approved by the Research Ethical Committee affiliated with the Brazilian Ministry of Health (CAAE-59488716.1.1001.5269). All procedures performed in this study were in accordance with the ethical standards specified in the World Medical Association Declaration of Helsinki. Informed consent was obtained for all patients and relatives for this study and the publication of images.

\section{Conflict of Interest Statement}

The authors have no conflicts of interest to declare.

\section{Funding Sources}

This research was partially funded by Conselho Nacional de Desenvolvimento Científico e Tecnológico (CNPq), Brasil, 440614/2016-3, 424494/2016-7, and 310772/2017-6; Coordenação 
de Pessoal de Nível Superior (CAPES), 88,881.130724/2016-01, and 8882.331372/2019-01 (grant to V.F.C.); Fundação Carlos Chagas Filho de Amparo à Pesquisa do Estado do Rio de Janeiro (FAPERJ), E-26/202.617/2019; Instituto Nacional de Genética Médica Populacional (INAGEMP - CNPq), 465549/2014-4 and 381592/2018-9 (grant to D.M.).

\section{Author Contributions}

I.M.O., V.F.d.C., C.P.S.: study design. V.F.d.C.: manuscript preparation. I.M.O., V.F.d.C., D.M.: revision of the manuscript. V.F.d.C., D.M., I.M.O.: data analysis. F.M.d.C., D.P.C, M.M.D.-R., J.L.Jr, V.C., R.S.H, J.C.L.L., M.T.S., M.P.A.d.S., P.B., A.M.B., L.C.S.d.S, P.B., P.S.C., L.S.M.B., I.M.O.: patient recruitment, clinical examination, and data collection. V.F.d.C., C.P.S.: laboratory work. I.M.O.: grant application, receipt of the funding, and supervision of the research.

\section{References}

Aruga J. The role of Zic genes in neural development. Mol Cell Neurosci. 2004;26(2):205-21.

Barratt KS, Arkell RM. ZIC2 in Holoprosencephaly. Adv Exp Med Biol. 2018;1046:269-99.

Belloni E, Muenke M, Roessler E, Traverso G, Siegel-Bartelt J, Frumkin A, et al. Identification of sonic hedgehog as a candidate gene responsible for holoprosencephaly. Nat Genet. 1996; 14(3):353-6.

Bosanac I, Maun HR, Scales SJ, Wen X, Lingel A, Bazan JF, et al. The structure of SHH in complex with HHIP reveals a recognition role for the Shh pseudo active site in signaling. Nat Struct Mol Biol. 2009;16(7):691-7.

Brown LY, Odent S, David V, Blayau M, Dubourg C, Apacik C, et al. Holoprosencephaly due to mutations in ZIC2: alanine tract expansion mutations may be caused by parental somatic recombination. Hum Mol Genet. 2001;10(8): 791-6.

Brown SA, Warburton D, Brown LY, Yu CY, Roeder ER, Stengel-Rutkowski S, et al. Holoprosencephaly due to mutations in ZIC2, a homologue of Drosophila odd-paired. Nat Genet. 1998;20(2):180-3.

Castilla EE, Orioli IM. ECLAMC: The LatinAmerican Collaborative Study of Congenital Malformations. Community Genet. 2004; 7(2-3):76-94.

Chen R, Davydov EV, Sirota M, Butte AJ. Nonsynonymous and synonymous coding SNPS show similar likelihood and effect size of human disease association. PLoS One. 2010; 5(10):e13574.

Cohen MM, Shiota K. Teratogenesis of holoprosencephaly. Am J Med Genet. 2002;109(1):115.

Cohen MM. Perspectives on holoprosencephaly: Part III. Spectra, distinctions, continuities, and discontinuities. Am J Med Genet. 1989a; 34(2):271-88

Cohen MM. Perspectives on holoprosencephaly: Part I. Epidemiology, genetics, and syndromology. Teratology. 1989b;40(3):211-35.

Darty K, Denise A, Ponty Y. VARNA: Interactive drawing and editing of the RNA secondary structure. Bioinformatics. 2009;25(15):1974-5.

DeMyer W, Zeman W, Palmer CG. The face predicts the brain: Diagnostic significance of median facial anomalies for Holoprosencephaly (Arhinencephaly). Pediatrics. 1964;34:25663.
Domené S, Roessler E, El-Jaick KB, Snir M, Brown JL, Vélez JI, et al. Mutations in the human SIX3 gene in holoprosencephaly are loss of function. Hum Mol Genet. 2008;17(24):3919-28.

Dubourg C, Lazaro L, Pasquier L, Bendavid C, Blayau M, Le Duff F, et al. Molecular screening of SHH, ZIC2, SIX3, and TGIF genes in patients with features of holoprosencephaly spectrum: Mutation review and genotypephenotype correlations. Hum Mutat. 2004; 24(1):43-51.

Dubourg C, Bendavid C, Pasquier L, Henry C, Odent S, David V. Holoprosencephaly. Orphanet J Rare Dis. 2007;2(1):1-14.

Dubourg C, Carré W, Hamdi-Rozé H, Mouden C, Roume J, Abdelmajid B, et al. Mutational Spectrum in Holoprosencephaly Shows That FGF is a New Major Signaling Pathway. Hum Mutat. 2016;37(12):1329-39.

Dubourg C, Kim A, Watrin E, de Tayrac M, Odent S, David V, et al. Recent advances in understanding inheritance of holoprosencephaly. Am J Med Genet C Semin Med Genet. 2018;178(2):258-69.

Dubourg C, Lazaro L, Blayau M, Pasquier L, Durou MR, Odent S, et al. Genetic study of holoprosencephaly (in French). Ann Biol Clin (Paris). 2003;61(6):679-87.

El-Jaick KB, Brunoni D, Castilla EE, Moreira MA, Orioli IM. SHH Ile111Asp in alobar holoprosencephaly in a proposita, whose mother had only a solitary median maxillary incisor. Am J Med Genet A. 2005;136A(4):345.

El-Jaick KB, Powers SE, Bartholin L, Myers KR, Hahn J, Orioli IM, et al. Functional analysis of mutations in TGIF associated with holoprosencephaly. Mol Genet Metab. 2007;90(1):97-111.

Fernandes M, Gutin G, Alcorn H, McConnell SK, Hébert JM. Mutations in the BMP pathway in mice support the existence of two molecular classes of holoprosencephaly. Development. 2007;134(21):3789-94.

Fu XD, Ares M. Context-dependent control of alternative splicing by RNA-binding proteins. Nat Rev Genet. 2014;15(10):689-701.

Geng X, Acosta S, Lagutin O, Gil HJ, Oliver G. Six 3 dosage mediates the pathogenesis of holoprosencephaly. Development. 2016; 143(23):4462-73.

Gripp KW, Wotton D, Edwards MC, Roessler E, Ades L, Meinecke P, et al. Mutations in TGIF cause holoprosencephaly and link NODAL signalling to human neural axis determination. Nat Genet. 2000;25(2):205-8.

Hamid R, Patterson J, Brandt SJ. Genomic structure, alternative splicing and expression of TG-interacting factor, in human myeloid leukemia blasts and cell lines. Biochim Biophys Acta. 2008;1779(5):347-55.

Hong M, Srivastava K, Kim S, Allen BL, Leahy DJ, $\mathrm{Hu} \mathrm{P}$, et al. BOC is a modifier gene in holoprosencephaly. Hum Mutat. 2017;38(11): 1464-70.

Hong M, Krauss RS. Modeling the complex etiology of holoprosencephaly in mice. Am J Med Genet C Semin Med Genet. 2018;178(2):140-50.

Hong S, Hu P, Marino J, Hufnagel SB, Hopkin RJ, Toromanović A, et al. Dominant-negative kinase domain mutations in FGFR1 can explain the clinical severity of Hartsfield syndrome. Hum Mol Genet. 2016;25(10):1912-22.

Hong S, Hu P, Roessler E, Hu T, Muenke M. Lossof-function mutations in FGF8 can be independent risk factors for holoprosencephaly. Hum Mol Genet. 2018;27(11):1989-98.

Hughes JJ, Alkhunaizi E, Kruszka P, Pyle LC, Grange DK, Berger SI, et al. Loss-of-Function Variants in PPP1R12A: From Isolated Sex Reversal to Holoprosencephaly Spectrum and Urogenital Malformations. Am J Hum Genet. 2020;106(1):121-8

Johnson CY, Rasmussen SA. Non-Genetic Risk Factors for Holoprosencephaly. Am J Med Genet C Semin Med Genet. 2010;154C(1):73-85.

Keaton A A, Solomon B D, Kauvar E F, El-Jaick K B, Gropman A L, Zafer Y, et al. TGIF Mutations in Human Holoprosencephaly: Correlation between Genotype and Phenotype. Mol Syndromol. 2010;1(5):211-222.

Kerr K, McAneney H, Smyth LJ, Bailie C, McKee $\mathrm{S}, \mathrm{McKnight}$ AJ. A scoping review and proposed workflow for multi-omic rare disease research. Orphanet J Rare Dis. 2020;15(1): 107.

Kim A, Savary C, Dubourg C, Carré W, Mouden $\mathrm{C}$, et al. Integrated clinical and omics approach to rare diseases: Novel genes and oligogenic inheritance in holoprosencephaly. Brain. 2019;142(1):35-49.

Kim A, Le Douce J, Diab F, Ferovova M, Dubourg $\mathrm{C}$, Odent $\mathrm{S}$, et al. Synonymous variants in holoprosencephaly alter codon usage and impact the Sonic Hedgehog protein. Brain. 2020; 143(7):2027-38. 
Kruszka P, Hart RA, Hadley DW, Muenke M, Habal MB. Expanding the phenotypic expression of Sonic Hedgehog mutations beyond holoprosencephaly. J Craniofac Surg. 2015;26(1):3-5.

Kruszka P, Berger SI, Casa V, Dekker MR, Gaesser J, Weiss K, et al. Cohesin complex-associated holoprosencephaly. Brain. 2019;142(9): 2631-43.

Lacbawan F, Solomon BD, Roessler E, El-Jaick K, Domené S, Vélez JI, et al. Clinical spectrum of SIX3-associated mutations in holoprosencephaly: correlation between genotype, phenotype and function. J Med Genet. 2009;46(6):389-98.

Landrum MJ, Lee JM, Benson M, Brown GR, Chao C, Chitipiralla S, et al. ClinVar: improving access to variant interpretations and supporting evidence. Nucleic Acids Res. 2018; 46(D1):D1062-7.

Maity T, Fuse N, Beachy PA. Molecular mechanisms of Sonic hedgehog mutant effects in holoprosencephaly. Proc Natl Acad Sci USA. 2005;102(47):17026-31.

Martinez AF, Kruszka PS, Muenke M. Extracephalic manifestations of nonchromosomal, nonsyndromic holoprosencephaly. Am J Med Genet C Semin Med Genet. 2018;178(2):246-57.

Matsunaga E, Shiota K. Holoprosencephaly in human embryos: Epidemiologic studies of 150 cases. Teratology. 1977;16(3):261-72.

Maurus D, Harris WA. Zic-associated holoprosencephaly: zebrafish Zicl controls midline formation and forebrain patterning by regulating Nodal, Hedgehog, and retinoic acid signaling. Genes Dev. 2009;23(12):1461-73.

McCabe MJ, Gaston-Massuet C, Tziaferi V, Gregory LC, Alatzoglou KS, Signore M, et al. Novel FGF8 mutations associated with recessive holoprosencephaly, craniofacial defects, and hypothalamo-pituitary dysfunction. J Clin Endocrinol Metab. 2011;96(10):E1709-18.

Mercier S, Dubourg C, Garcelon N, CampilloGimenez B, Gicquel I, Belleguic M, et al. New findings for phenotype-genotype correlations in a large European series of holoprosencephaly cases. J Med Genet. 2011;48(11):752-60.

Miller SA, Dykes DD, Polesky HF. A simple salting out procedure for extracting DNA from human nucleated cells. Nucleic Acids Res. 1988;16(3):1215.

Mouden C, Dubourg C, Carré W, Rose S, Quelin C, Akloul L, et al. Complex mode of inheritance in holoprosencephaly revealed by whole exome sequencing. Clin Genet. 2016;89(6):659-68.

Nanni L, Ming JE, Bocian M, Steinhaus K, Bianchi DW, Die-Smulders C, et al. The mutational spectrum of the Sonic Hedgehog gene in holoprosencephaly: SHH mutations cause a significant proportion of autosomal dominant holoprosencephaly. Hum Mol Genet. 1999; 8(13):2479-88

Nanni L, Ming JE, Du Y, Hall RK, Aldred M, Bankier A, et al. SHH mutation is associated with solitary median maxillary central incisor: a study of 13 patients and review of the literature. Am J Med Genet. 2001;102(1):1-10.

Odent S, Le Marec B, Munnich A, Le Merrer M, Bonaïti-Pellié $\mathrm{C}$. Segregation analysis in non- syndromic holoprosencephaly. Am J Med Genet. 1998;77(2):139-43.

Ohno K, Takeda JI, Masuda A. Rules and tools to predict the splicing effects of exonic and intronic mutations. Wiley Interdiscip Rev RNA. 2018;9(1).

Orioli IM, Castilla EE. Epidemiology of holoprosencephaly: Prevalence and risk factors. Am J Med Genet C Semin Med Genet. 2010; $154 \mathrm{C}(1): 13-21$

Orioli IM, Castilla EE, Ming JE, Nazer J, Burle De Aguiar MJ, Llerena JC, et al. Identification of novel mutations in SHH and ZIC2 in a South American (ECLAMC) population with holoprosencephaly. Hum Genet. 2001;109(1):1-6.

Pagani F, Raponi M, Baralle FE. Synonymous mutations in CFTR exon 12 affect splicing and are not neutral in evolution. Proc Natl Acad Sci USA. 2005;102(18):6368-72.

Paulussen AD, Schrander-Stumpel CT, Tserpelis DC, Spee MK, Stegmann AP, Mancini GM, et al. The unfolding clinical spectrum of holoprosencephaly due to mutations in $\mathrm{SHH}$, ZIC2, SIX3 and TGIF genes. Eur J Hum Genet. 2010;18(9):999-1005.

Pechmann S, Frydman J. Evolutionary conservation of codon optimality reveals hidden signatures of cotranslational folding. Nat Struct Mol Biol. 2013;20(2):237-43.

Presnyak V, Alhusaini N, Chen YH, Martin S, Morris N, Kline N, et al. Codon optimality is a major determinant of mRNA stability. Cell. 2015;160(6):1111-24.

Ribeiro LA, El-Jaick KB, Muenke M, Richieri-costa A. SIX3 Mutations with Holoprosencephaly. Am J Med Genet A. 2006;140(23):2577-83.

Ribeiro LA, Roessler E, Hu P, Pineda-Alvarez DE, Zhou N, Jones M, et al. Comparison of Mutation Findings in ZIC2 between Microform and Classical Holoprosencephaly in a Brazilian Cohort. Birth Defects Res Part A Clin Mol Teratol. 2012;94(11):912-7.

Richieri-Costa A, Ribeiro LA. Holoprosencephalylike phenotype: clinical and genetic perspectives. Am J Med Genet A. 2006;140(23):2587-93.

Roelink H. Sonic Hedgehog is a member of the $\mathrm{Hh} / \mathrm{DD}$-peptidase family that spans the eukaryotic and bacterial domains of life. J Dev Biol. 2018;6(2): 12 .

Roessler E, Belloni E, Gaudenz K, Jay P, Berta P, Scherer SW, et al. Mutations in the human sonic hedgehog gene cause holoprosencephaly. Nat Genet. 1996;14(3):357-60.

Roessler E, El-Jaick KB, Dubourg C, Vélez JI, Solomon BD, Pineda-Alvarez DE, et al. The mutational spectrum of holoprosencephaly-associated changes within the SHH gene in humans predicts loss-of-function through either key structural alterations of the ligand or its altered synthesis. Hum Mutat. 2009a;30(10):E921-35.

Roessler E, Lacbawan F, Dubourg C, Paulussen A, Herbergs J, Hehr U, et al. The full spectrum of holoprosencephaly-associated mutations within the ZIC2 gene in humans predicts lossof-function as the predominant disease mechanism. Hum Mutat. 2009b;30(4):E54154.
Roessler E, Vélez JI, Zhou N, Muenke M. Utilizing prospective sequence analysis of SHH, ZIC2, SIX3 and TGIF in holoprosencephaly probands to describe the parameters limiting the observed frequency of mutant genexgene interactions. Mol Genet Metab. 2012;105(4): 658-64.

Roessler E, Hu P, Marino J, Hong S, Hart R, Berger $\mathrm{S}$, et al. Common genetic causes of holoprosencephaly are limited to a small set of evolutionarily conserved driver genes of midline development coordinated by TGF- $\beta$, hedgehog, and FGF signaling. Hum Mutat. 2018a;39(10):1416-27.

Roessler E, Hu P, Muenke M. Holoprosencephaly in the genomics era. Am J Med Genet C Semin Med Genet. 2018b;178(2):165-74.

Savastano CP, El-Jaick KB, Costa-lima MA, Abath CM, Bianca S, Cavalcanti DP, et al. Molecular analysis of holoprosencephaly in South America. Genet Mol Biol. 2014;37(1 Suppl 1):250-62.

Singh S, Tokhunts R, Baubet V, Goetz JA, Huang ZJ, Schilling NS, et al. Sonic hedgehog mutations identified in holoprosencephaly patients can act in a dominant negative manner. Hum Genet. 2009;125(1):95-103.

Solomon BD, Lacbawan F, Mercier S, Clegg NJ, Delgado MR, Rosenbaum K, et al. Mutations in ZIC2 in human holoprosencephaly: Description of a Novel ZIC2 specific phenotype and comprehensive analysis of 157 individuals. J Med Genet. 2010;47(8): 513-24.

Solomon BD, Bear KA, Wyllie A, Keaton AA, Dubourg C, David V, et al. Genotypic and phenotypic analysis of 396 individuals with mutations in Sonic Hedgehog. J Med Genet. 2012; 49(7):473-9.

Soukarieh O, Gaildrat P, Hamieh M, Drouet A, Baert-Desurmont S, Frébourg T, et al. Exonic Splicing Mutations Are More Prevalent than Currently Estimated and Can Be Predicted by Using In Silico Tools. PLoS Genet. 2016; 12(1):e1005756.

Stergachis AB, Haugen E, Shafer A, Fu W, Vernot $\mathrm{B}$, Reynolds A, et al. Exonic transcription factor binding directs codon choice and affects protein evolution. Science. 2013;342(6164): 1367-72.

Traiffort E, Dubourg C, Faure H, Rognan D, Odent S, Durou MR, et al. Functional characterization of Sonic hedgehog mutations associated with holoprosencephaly. J Biol Chem. 2004;279(41):42889-97.

Wallis D, Muenke M. Mutations in Holoprosencephaly. Hum Mutat. 2000;16(2):99-108.

Wallis DE, Roessler E, Hehr U, Nanni L, Wiltshire $\mathrm{T}$, Richieri-Costa A, et al. Mutations in the homeodomain of the human SIX3 gene cause holoprosencephaly. Nat Genet. 1999;22(2): 196-8.

Wotton D, Taniguchi K. Functions of TGIF homeodomain proteins and their roles in normal brain development and holoprosencephaly. Am J Med Genet C Semin Med Genet. 2018;178(2):128-39. 